J. Indones. Math. Soc.

Special Edition (2011), pp. 123-131.

\title{
TOTALLY MAGIC INJECTIONS
}

\author{
W. D. WALLis
}

Southern Illinois University, Carbondale, Illinois, USA

\begin{abstract}
A labelling is a mapping whose domain is some set of graph elements - the set of vertices, for example, or the set of all vertices and edges - and whose range is a set of positive integers. In particular, if the labels associated with any edge - the label on the edge itself, and those on its endpoints - always add to the same sum, the labeling, and the graph possessing it, is called magic. A related concept, a vertex-magic total labeling, is one in which the sum of the label on any vertex with the labels on the edges containing it is always constant. A labeling which has both the vertex-magic and edge-magic properties (usually with two different constants) is called totally magic, as is a graph possessing such a labeling. In this paper we survey what is known about totally magic graphs and an important generalization.

Key words: Magic, vertex-magic total labeling, totally magic.
\end{abstract}

2000 Mathematics Subject Classification: $05 \mathrm{C} 78$.

Received: 09-08-2011, revised: 09-09-2011, accepted: 04-12-2012. 